\title{
MONITORING DYNAMICS IN TIME OF FOREST VERTICAL STRUCTURE WITH MULTIBASELINE POLINSAR DATA
}

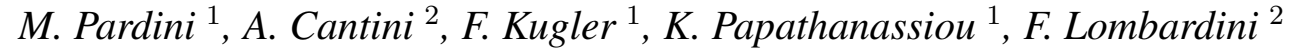 \\ ${ }^{1}$ German Aerospace Center (DLR) - Microwave and Radar Institute (HR) - Wessling, Germany \\ ${ }^{2}$ University of Pisa - Department of Information Engineering - Pisa, Italy
}

\begin{abstract}
Synthetic aperture radar (SAR) waves have the capability of penetrating into forest volumes. Multibaseline (MB) polarimetric-interferometric SAR (PolInSAR) systems can retrieve information about the vertical distribution of the radar backscattered power and to relate it to the physical 3-D forest structure of the vegetation.

Radar backscattering from forests can change in time due to different weather conditions, to seasonality, and to logging or disturbances. In this paper, experiments with L-band and $\mathrm{X}$-band data are presented for a first characterization of the related effects in the estimated 3-D forest structure parameters.
\end{abstract}

Index Terms - Synthetic Aperture Radar (SAR), polarimetry, tomography, forest structure, forest dynamics.

\section{INTRODUCTION}

Synthetic aperture radar (SAR) data possess penetration capabilities into forests, and they have been demonstrated to be sensitive to their vertical structure at both low (P-/L-band) and high (X-band) frequencies.

A significant advance in the analysis of forest vertical structure came with the coherent combination of polarimetry with interferometry (Pol-InSAR) [1]. Polarimetry can interprete different scattering mechanisms, while interferometry can locate them in height. In this way, the inversion of multilayer scattering models has been made possible, with applications like forest height estimation, classification, and biomass estimation. The possibility of interpreting multiple scattering components in height in a continuous profile of the backscattered power has been demonstrated also with SAR Tomography (TomoSAR) [2], which in principle exploits only baseline diversity, but it can be greatly improved by coupling it with polarimetry. Recent research on TomoSAR for forest applications has been focused mainly on the experimentation of techniques (model-based and not) able to overcome the limits intrinsic of a simple Fourier-based focusing (see e.g. [2]-[4]).

In general, the 3-D distribution of the backscattered power can change due to different weather conditions (due to changes in the dielectric properties of the vegetation layers), to seasonality (leaf-on/leaf-off condition), and to logging or disturbances. The characterization of these changes in TomoSAR profiles is essential for understanding the link between the 3-D backscattered power and the physical forest structure. This link depends on frequency and its establishment is fundamental towards potential TomoSAR-based applications, especially for documenting forest ecosystem changes. In this work, first results are reported in order to analyze the changes on forest tomograms primarily due to weather and seasonal effects. Experiments have been carried out mostly by processing a multitemporal-multibaseline (MB) L-band airborne data set acquired by the DLR ESAR platform over the forest site of Traunstein (South of Germany). X-band potentials have also been evaluated by processing MB TanDEM-X data.

\section{WEATHER AND SEASONAL EFFECTS AT L-BAND}

The repeat-pass DLR E-SAR system acquired fully polarimetric and interferometric L-band SAR data over the Traunstein forest site (South of Germany) in the frame of the DLR TempoSAR campaign in 2008 and 2009. Baselines and weather classification for the acquisitions selected in this analysis are reported in Tab. $1^{1}$.

Concerning the 3-D TomoSAR focusing, for the sake of simplicity, in this work we resort to the adaptive beam forming (i.e. Capon spectral estimator, abbreviated ABF), in order to achieve height super-resolution and sidelobe rejection. Moreover, to get acceptable accuracy and radiometric fidelity, profiles have been calculated by averaging approximately 100 independent looks. In terms of adjacent range and azimuth pixels, this corresponds to a square multilook cell with side measuring $15 \mathrm{~m}$. In addition, structure parameters (here ground-to-volume power ratio and phase center height) have been estimated by means of MB techniques (see [6] for details). The comparison of the spring and autumn profiles has been carried out after interpolation to a common baseline distribution.

\footnotetext{
${ }^{1}$ A full description of the data set with detailed weather data can be found in [5].
} 


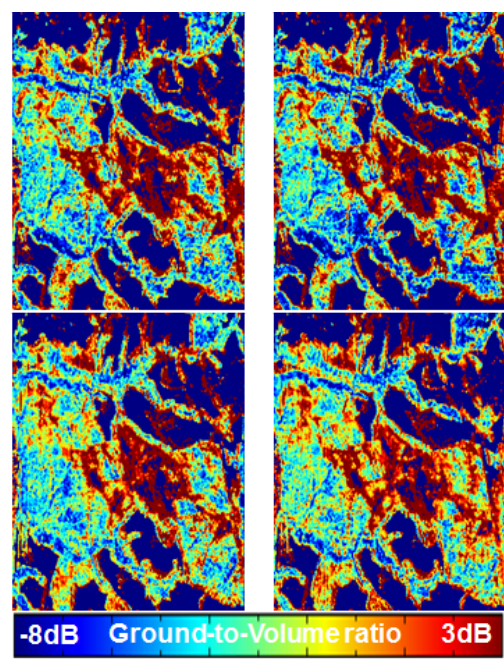

(a) Ground-to-Volume power ratio

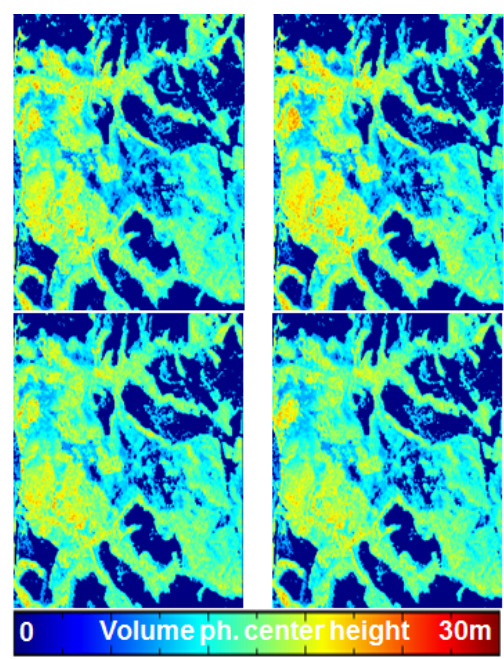

(b) Phase center height

Fig. 1. Traunstein forest site: estimated parameters in the range (horizontal) - azimuth plane, $\mathrm{HH}$ channel. (a) and (b), Top row: dry (left) and rainy day (right); bottom row: spring (left), autumn (right).

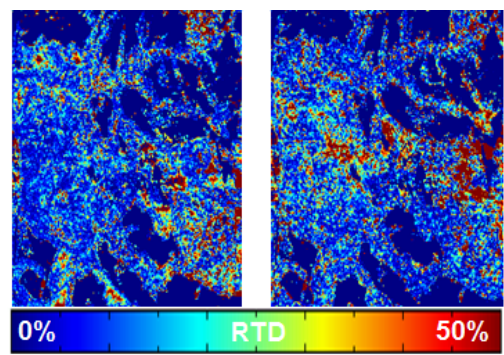

Fig. 2. Traunstein forest site: estimated RTD of the ABF profiles in the range (horizontal) - azimuth plane, HH channel. Left: dry day vs wet day; right: spring vs autumn.

\begin{tabular}{ccc}
\hline \hline Acq. day & Hor. baselines $(\mathrm{m})$ & Conditions \\
\hline $10 / 06 / 2008$ & $-15,-5,0,5,10$ & Dry \\
$12 / 06 / 2008$ & $-15,-5,0,5,10$ & Wet \\
$11 / 05 / 2009$ & $-15,-5,-10,0,5,10,15$ & Dry \\
$28 / 10 / 2009$ & $0,5,10,15$ & Dry \\
\hline \hline
\end{tabular}

Table 1. TempoSAR 2008/2009 acquisitions selected for the analysis. Baselines are measured with respect to the master image. Conditions (wet/dry) are defined based on the precipitation data.

The radar backscatter from forests is mostly related to the dielectric constant of the tree tissues, that depends strongly on their water content. After a rain event the water content inside leaves and trunk increases. From an electromagnetic point of view, it is reasonable to expect $i$ ) a decrease of the ground-to-volume power ratio, (ii) an increase of the phase center height of the TomoSAR reflectivity profile, and (iii) a general change in the backscattered power of each single layer. On the other hand, seasonal effects in temperate regions are mainly characterized by the absence of leaves in autumn and winter, and therefore a reduction of water transpiration and quantity of water in the tree trunks. In radar terms, this means an increase of penetration into the vegetation volume.

The ground-to-volume power ratios $\mu$ estimated in a portion of the Traunstein forest measuring around $1 \mathrm{Km}$ in slant range and $2 \mathrm{Km}$ in azimuth are shown in Fig. 1(a) for all of the acquisition dates in the $\mathrm{HH}$ polarization channel. In the top row of Fig. 1(a), it is possible to observe that in the wet day $\mu$ tends to decrease as expected, although the variation depends on the forest stand. Considering forest stands with a height of around $30 \mathrm{~m}$, it has been measured an average decrease of $1.45 \mathrm{~dB}$ for the stand with the lowest biomass (300t/ha). At the increase of biomass, the decrease of $\mu$ is still observable, although with a a higher dispersion. Seasonal changes of $\mu$ are visible in the bottom row of Fig. 1(a). It is apparent that, differently from the case of weather variations, in autumn $\mu$ is almost uniformly higher than in spring, and the increase amounts to $3 \mathrm{~dB}$ in average, with a maximum of even $5 \mathrm{~dB}$. This is visible independently of the biomass level. In addition, it has also been seen that variations can depend on the species. Indeed, for broad-leaved stands the ground power increases by $2 \mathrm{~dB}$ (average) in autumn with respect to spring, while for conifers the increase does not reach $1 \mathrm{~dB}$. In addition, volume powers of broad-leaved stands decrease of about $1 \mathrm{~dB}$ while for conifer stands they are almost constant.

The variations of the phase center heights are shown in Fig. 1(b). From the top row, it can be observed that after the rainfall the phase center height increases of around $3 \mathrm{~m}$ in average. Higher values can be obtained depending on the forest stand. The bottom row, instead, shows that the change of season from spring to autumn causes a general decrease of the phase center height (indicating a higher penetration), although to a lower extent with respect to the increase observed 
with the change of weather conditions.

From Fig. 1 it is also apparent that a decrease of $\mu$ not necessarily corresponds to an increase of the phase center height, and vice versa. This may be an indication that a rainfall causes changes of the entire TomoSAR structure. To get further insights, we evaluated the changes of the entire ABF tomogram in terms of the relative total difference (RTD) of the profiles, defined as:

$$
\xi=\frac{\sum_{m=1}^{M}\left[f_{1}\left(z_{m}\right)-f_{2}\left(z_{m}\right)\right]^{2}}{\left.\sum_{m=1}^{M} f_{1}^{2}\left(z_{m}\right)\right]},
$$

being $f_{1}(z)$ and $f_{2}(z)$ the two profiles under comparison, $z_{m}$, $m=1, \ldots, M$ the heights at which they are sampled and $M$ the total number of samples between the ground and the canopy top. The RTD resulting from the comparison of the wet and dry data sets and the spring and autumn data sets are reported in Fig. 2. Changing from a dry to a wet day, the RTD can reach values around the $15 \%$, though the RTD can be higher for some stands. Conversely, the change of season affects much more the RTD, which now assumes values higher than $25 \%$ in many stands. Experiments are currently ongoing in order to further understand these profile variations.

\section{RESULTS WITH TANDEM-X DATA}

The single-pass interferometric mission TanDEM-X allows to acquire and analyse $\mathrm{X}$-band PolInSAR data without the disturbing effects of temporal decorrelation. Rather suprisingly, recent TanDEM-X experiments for forest applications have demonstrated penetration capabilities of X-band radar signals into volumes, depending also on their density. In this Section, we show a few first results that demonstrate some potentials of X-band to document forest changes.

\subsection{Seasonal changes}

A simple way to evaluate the penetration depth is to resort to the maximum polarimetric phase difference $\Delta \phi$, which indicates the maximum variation of the interferometric phase obtained by changing polarization channel.

It is expected that repeated measurements of $\Delta \phi$ can be used to detect seasonal changes, as they are connected with the variation of the penetration depth. This potential has been evaluated here by means of a multitemporal TanDEM-X data set over the decidous riverine forest of Plattling (South of Germany). For this purpose, a sequence of three dual-pol (HH, VV) stripmap acquisitions covering the foliation period (from the middle of April 2011 until the middle of May 2011) was available, all of them with an interferometric height of ambiguity around $30 \mathrm{~m}$. For these three acquisitions, the corresponding $\Delta \phi / k_{z}$ were calculated, and they are reported in Fig. 3 in meters after rescaling by the vertical wavenumber $k_{z}$. The first acquisition (12/04/2011) refers to the leaf-off stage, and $\Delta \phi / k_{z}$ assumes values larger than $4 \mathrm{~m}$ nearly all

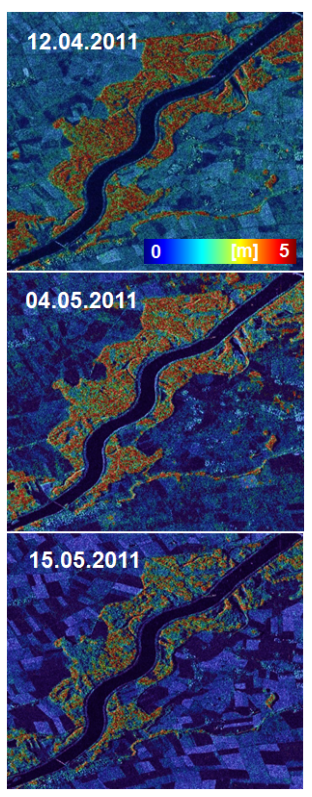

Fig. 3. Plattling forest site: maximum polarimetric phase difference in the range-azimuth plane for three dates in the foliation process.

over the forested area. In the second acquisition (04/05/2011), the foliation already started and $\Delta \phi / k_{z}$ decreases accordingly. In the last acquisition (15/05/2011), the foliation is almost completed and $\Delta \phi / k_{z}$ rarely reaches values larger than $4 \mathrm{~m}$. As expected, the increase of the foliage in the canopy progressively decreases the penetration capability of X-band.

As a final comment, is it worth remarking that the sensitivity to seasonal changes has an impact also in forest height estimation from TanDEM-X data. Indeed, depending on the forest environment, the change of penetration depth (for instance from summer to winter) may in some case correspond to the presence of a more or less powerful ground scattering contribution, which could drive the choice of the most suitable inversion scheme. These aspects have been fully investigated and quantified in [7].

\subsection{Potentials of $\mathrm{X}$-band TomoSAR}

One can also explore the possibility of retrieving a full 3D profile of the backscattered power starting from multiple single-pass PolInSAR acquisitions with different baselines. As suggested in [8]-[9], this objective can be accomplished in a coherence tomographic framework. In particular, supposing the Random-Volume-over-Ground model to be valid, it is possible to retrieve a full MB-polarimetric data covariance matrix by means of a baseline interpolation step followed by algebraic techniques [9]. In this way, tomograms can be derived by applying classical high resolution spectral estimation techniques.

The sample ABF tomographic slice shown in Fig. 4 has 


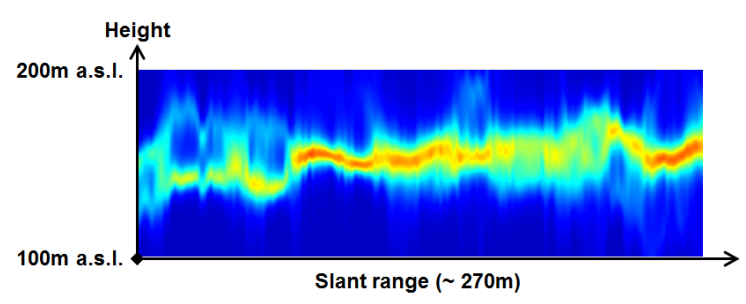

Fig. 4. Tapajos forest site: ABF tomogram in the slant rangeheight plane for a fixed azimuth coordinate, $\mathrm{HH}$ channel. Color scale of the backscattered power from blue (minimum) to red (maximum).

been obtained by processing a portion of a range line (at fixed azimuth) of a MB time series of TanDEM-X data over the Amazonian Tapajos national forest. The Tapajos test site is located in the Amazon basin in northern Brazil beside the Tapajos River. The region of Tapajos is fairly flat and predominantly vegetated by undisturbed primary rainforest. Five TanDEM-X dual-pol (HH/VV) interferograms were available for processing. They had been acquired between July and November 2011, i.e. during the dry season, with height of ambiguity ranging almost uniformly from around $50 \mathrm{~m}$ to $150 \mathrm{~m}$ and incidence angle of 40deg. It has been verified that the each acquisition was performed in almost uniform weather conditions. Rather unexpectedly, the tomogram of Fig. 4 shows a variety of profiles (even with two layers), that seem to be related not only to the penetration depth, but also to a full 3-D (horizontal and vertical) vegetation structure. This result, although still in a validation stage, clearly indicates that $\mathrm{X}$-band has the potential of distinguishing between different 3-D structures, and therefore it is expected to be sensitive also to their changes, e.g. reduction of density due to logging activities.

\section{CONCLUSIONS}

The experiments reported in this paper have shown that MB PolInSAR acquisitions allow to observe weather and seasonal changes of the 3-D distribution of the forest radar backscattering, even with a low number of tracks and a moderate Rayleigh resolution in height. L-band data are sensitive to volume propagation, and changes have been observed of the whole vertical power profile. The (unexpectedly high) penetration of short-wavelength X-band signals was demonstrated to be sensitive e.g. to the foliation process, and to allow even the tomographic profiling of a tropical forest, with potentials in detecting density changes, for instance due to deforestation. However, the question is still open if $\mathrm{X}$-band penetration corresponds to propagation in the vegetation volume or partially through gaps, that become relevant at high resolution. Although still in a first stage, the experiments reported in this paper highlight that proper methodologies have to be set up for extracting information about the physical structure and of its changes that for instance are robust to weather effects, taking into account the frequency-dependent mapping of vegetation components into radar backscattering.

\section{REFERENCES}

[1] S. Cloude, K. Papathanassiou, "Polarimetric SAR Interferometry", IEEE Trans. on Geosci. and Rem. Sensing, Vol. 36, No. 5, pp. 1551-1565, May 1998.

[2] A. Reigber, A. Moreira, "First Demonstration of Airborne SAR Tomography Using Multibaseline L-band Data”, IEEE Trans. on Geosci. and Rem. Sensing, Vol. 38, No. 5, pp. 2142-2152, May 2000.

[3] F. Lombardini, A. Reigber, "Adaptive Spectral Estimation for Multibaseline SAR Tomography with L-band Airborne data", Proc. of IEEE Int. Geosci. and Rem. Sensing Symposium (IGARSS), Toulouse, France, Jul. 2003.

[4] S. Tebaldini, "Single and Multipolarimetric SAR Tomography of Forested Areas: A Parametric Approach", IEEE Trans. on Geosci. and Rem. Sensing, Vol. 48, No. 5, pp. 2375-2387, May 2010.

[5] S.K. Lee, F. Kugler, K. Papathanassiou, et al., "Quantification of Temporal Decorrelation Effects at L-Band for Polarimetric SAR Interferometry Applications", IEEE Journal of Sel. Topics in Earth Obs. and Rem. Sensing, Vol. 6, No. 3, pp. 1351-1367, Jun. 2013.

[6] M. Pardini, K. Papathanassiou, "Sub-Canopy Topography Estimation: Experiments With Multibaseline SAR Data at L-Band", Proc. of IEEE Int. Geosci. and Rem. Sensing Symposium (IGARSS), Munich, Germany, Jul. 2012.

[7] F. Kugler, D. Schulze, I. Hajnsek, et al., "TanDEM-X Pol-InSAR Performance for Forest Height Estimation," IEEE Trans. on Geosci. and Rem. Sensing, Vol. 52, No. 10, pp. 6404-6422, Oct. 2014.

[8] F. Lombardini, M. Pardini, "Sector-Matched Interpolation for 3D SAR Correlative Imaging," Technical Report, Edizioni ETS, Pisa, Italy, Jul. 2008.

[9] M. Pardini, A. Toraño-Caicoya, F. Kugler, et al. "Estimating and Understanding Vertical Structure of Forests from Multibaseline TanDEM-X Pol-InSAR data", Proc. of IEEE Int. Geosci. and Rem. Sensing Symposium (IGARSS), Melbourne, Australia, Jul. 2013. 